\title{
A review of linear advanced current control techniques for grid connected PV inverters
}

\author{
Javier Monreal, Ignacio Benítez, Laura Moreno, Andrés Lluna and Inma Díaz \\ Department of Capital Goods and Automation \\ Instituto Tecnológico de la Energía (ITE) \\ Avenida Juan de la Cierva, 24 - Parque Tecnológico de Valencia - Paterna, 46980 Valencia (Spain) \\ Phone/Fax number: +0034 9613666 70, e-mail: javier.monreal@ite.es, ignacio.benitez@ite.es
}

\begin{abstract}
The following paper presents the work being done on the comparative design, simulation and experiments concerning advanced digital linear control techniques for power inverters. Based on an existing inverter topology being developed and implemented, with a conventional DSPprogrammed PI control, a research is done on more advanced linear control techniques that might improve the PI response, allowing at the same time a separate regulation of active and reactive power for electricity connection to the grid.
\end{abstract}

\section{Key words}

Inverter, isolation transformer, filter, linear control, dead beat, resonant, PI.

\section{Introduction}

The Energy Technological Institute (ITE) has as one of its objectives, to study how to improve energy efficiency in both industrial and domestic sectors. This implies not only loads, but also generation. In this framework, a power inverter is being built, designed to work either in isolated mode, or connected to the grid.

In this paper, a review of the main linear current control techniques for a $20 \mathrm{~kW}$ photovoltaic inverter, connected to the grid through an isolation transformer, is carried out. The inverter has been designed and implemented by ITE, as a modular hardware platform controlled by a digital signal processor (DSP).

The main objective of this project is to review, model, simulate and test control techniques developed to control the power flow inverter, at the level of the current control loop, with two objectives:

- Power Quality, i.e., reducing total harmonic distortion (THD) and ensuring an stable supply

- A separate regulation of active and reactive power.
The performances of classical and modern techniques are compared by simulation using the power electronics software PSIM.

Different control techniques have been applied, in a framework of cooperation to overcome the drawbacks that each one might present as a stand-alone configuration. Furthermore, different issues related with enhancing the performance of the inverter have been considered and gathered in the same design. These issues have been integrated based on the bibliography and the state of the art of grid connected inverters [1].

Since the inverter is connected through an isolation transformer, its influence has been evaluated and indeed has been included as a part of the global model to design the controllers.

The work being developed is currently at the simulation stage. However, the simulations have been carried out on the same electronic design where the previous PI controller was tuned to control the implemented inverter. The new control developments will be implemented in the inverter's digital signal processor (DSP), tested and compared.

\section{Objectives}

The aim of this paper is to compare the classic PI dq control performance of a $20 \mathrm{~kW}$ grid inverter already tuned, with other control techniques found in the literature, either alone or in a framework of cooperation among them to improve the overall performance of the inverter. The techniques being developed and simulated are the following:

- Discrete PI with decoupling.

- Dead beat control with integral action [2].

- Proportional - Resonant controller [3].

Furthermore, some aspects are wanted to be assessed, such as computational delay and uncertainty robustness in the value of the parameters of the inverter and grid. 
Other schemas of deadbeat control have also been implemented, such as a design with a plug-in repetitive controller [4], and a deadbeat cascaded control. The design of predictive observers to bear with computational delay has been carried out as well.

In the following paper, first the description and dynamic model of the inverter implemented by ITE are described, followed by the design and simulation of the aforementioned control techniques. A conclusions and future works section is also included.

\section{Inverter Description}

The inverter is a typical VSI (voltage-source inverter) configuration with a LC filter connected to the grid through an isolation transformer. The inverter's electronic schematic model design is depicted in Fig. 1, where a DC link is assumed to provide a constant $650 \mathrm{~V}$ dc voltage.

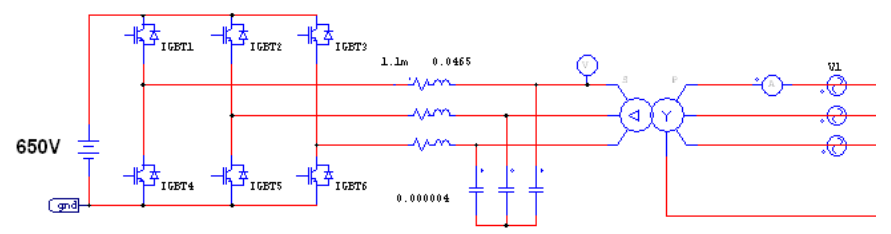

Fig. 1. Schematic model of the grid connected inverter with isolation transformer

\subsection{Theoretical model}

For the control design, an ASM (Average Switching Model) of the inverter filter and an estimation of the minimum grid impedance will be considered. As the minimum expected impedance seen by the inverter is provided by the equivalent impedance of the transformer, this is calculated looking from the inverter side towards the grid and including the equivalent circuit in the filter global model, as can be seen in Fig. 2.

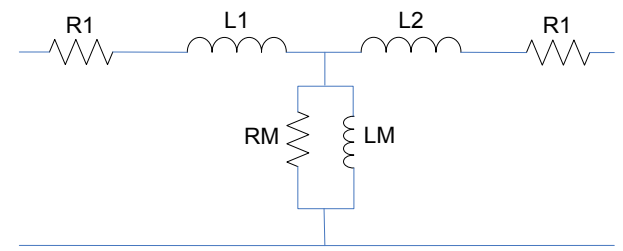

Fig. 2. Equivalent circuit of the isolation transformer.

The impedance seen by the inverter is usually larger than this because of the grid impedance, however this calculation provides the minimum impedance that can be expected [5]. In order to include the transformer into the dynamic global model, an assumption is made, considering that the transformer magnetization impedance $\left(\mathrm{Z}_{\mathrm{M}}\right)$ has a high value, so the simplified equivalent model of the transformer is an inductance $\left(\mathrm{L}_{\mathrm{T}}\right)$.
Thus the equivalent global model becomes an $\mathrm{L}_{\mathrm{f}} \mathrm{C}_{\mathrm{f}} \mathrm{L}_{\mathrm{T}}$ filter, which can be approximated by an $\mathrm{L}$ filter. This holds for frequencies lower than the resonance frequency of LCL filter, as can be seen from Fig. 3.

The inverter model has been obtained applying the $d-q$ transform [6] which expresses a three-phase, balanced system as a linear, two-phase approximation system, where $a c$ variables are converted to constant values.

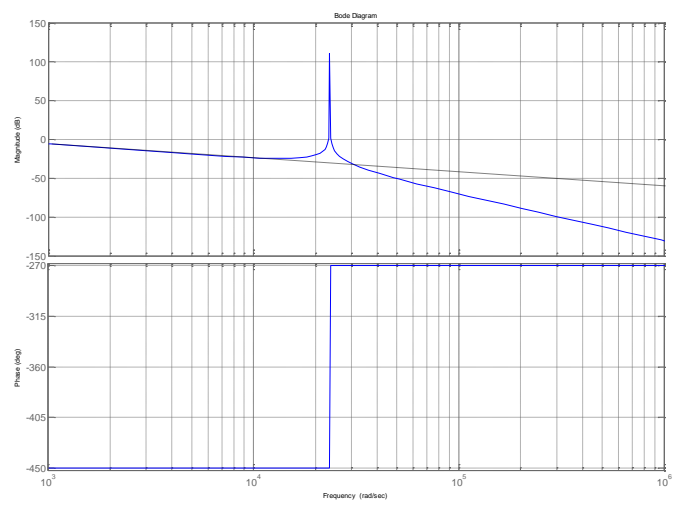

Fig. 3. Bode diagram of the equivalent filter.

The state space description of the filter average model, in terms of $\mathrm{d}-\mathrm{q}$ transformation, incorporating the lumped internal resistance $\mathrm{R}$, is given in Eq. 1 .

$$
\dot{x}=\frac{d}{d t}\left[\begin{array}{l}
i d \\
i q
\end{array}\right]=\underbrace{\left[\begin{array}{cc}
-\frac{R}{L} & w \\
-w & -\frac{R}{L}
\end{array}\right]}_{A}\left[\begin{array}{c}
i d \\
i q
\end{array}\right]+\underbrace{\left[\begin{array}{cc}
\frac{1}{L} & 0 \\
0 & \frac{1}{L}
\end{array}\right]}_{B 1}\left[\begin{array}{l}
u d \\
u q
\end{array}\right]+\underbrace{\left[\begin{array}{cc}
-\frac{1}{L} & 0 \\
0 & -\frac{1}{L}
\end{array}\right]}_{B 2}\left[\begin{array}{l}
v d \\
v q
\end{array}\right]
$$

\subsection{Discrete model}

The sample frequency was fixed to $12200 \mathrm{~Hz}$ in the digital implementation of the original control in the DSP. Since the sampling frequency is much higher than the frequency of the system, the Euler method can be used to discretize the model. The result can be observed in Eq. 2 .

$$
\left[\begin{array}{l}
i d(k+1) \\
i q(k+1)
\end{array}\right]=\underbrace{\left[\begin{array}{cc}
1-\frac{T_{S} R}{L} & T_{S} w \\
-T_{S} w & 1-\frac{T_{S} R}{L}
\end{array}\right]}_{\text {Ad }}\left[\begin{array}{l}
i d(k) \\
i q(k)
\end{array}\right]+\underbrace{\left[\begin{array}{cc}
\frac{T_{S}}{L} & 0 \\
0 & \frac{T_{S}}{L}
\end{array}\right]}_{B \text { Bid }}\left[\begin{array}{l}
u d(k) \\
u q(k)
\end{array}\right]+\underbrace{\left[\begin{array}{cc}
-\frac{T_{S}}{L} & 0 \\
0 & -\frac{T_{S}}{L}
\end{array}\right]}_{B 2 d} v \begin{array}{l}
v d(k) \\
v q(k)
\end{array}]
$$

\section{Controllers design}

Once the bibliographic study has been made, a total of three control designs have been selected to be implemented and tested at the inverter. The initial stage comprehends modeling and simulation of these control techniques applied to the inverter model. This work is detailed next. 


\subsection{Discrete PI with decoupling and compensation of grid voltage disturbance}

This control design is developed in the dq reference framework. In order to improve the performance of the inverter, decoupling the two axes, $\mathrm{d}$ and $\mathrm{q}$, becomes necessary. In addition, grid voltages $\mathrm{v}_{\mathrm{d}}$ and $\mathrm{v}_{\mathrm{q}}$ represent disturbance signals acting on the system (see Fig. 4). These voltages are measured in the system and thus serve to determine the angle of the rotating reference frame. Therefore, it is possible to directly compensate such disturbances. Considering the state space model of Eq. 2, decoupling $\mathrm{dq}$ axis and compensating grid voltages are very easy to incorporate into an equivalent control action with the corresponding terms described in Eq. 3.

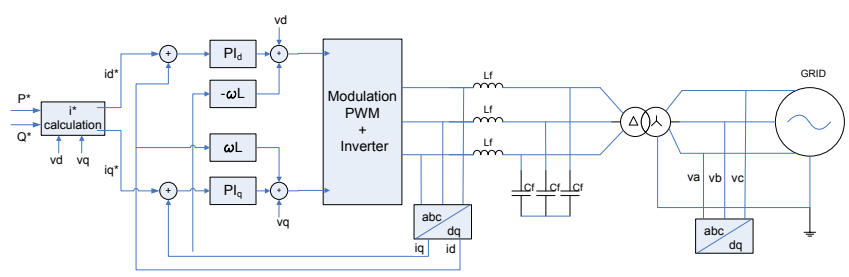

Fig. 4. Block diagram for decoupled PI control.

$$
\left[\begin{array}{l}
u_{d}^{\prime} \\
u_{q}^{\prime}
\end{array}\right]=\left[\begin{array}{cc}
0 & \omega L \\
-\omega L & 0
\end{array}\right]\left[\begin{array}{l}
i_{d}(k) \\
i_{q}(k)
\end{array}\right],-\left[\begin{array}{l}
v_{d} \\
v_{q}
\end{array}\right]+\left[\begin{array}{l}
u_{d} \\
u_{q}
\end{array}\right]
$$

Thus the equivalent transfer function of the system with respect to the equivalent control action for each of the two axes is a first order function, as written in Eq. 4.

$$
P(s)=\frac{1}{s L+R}
$$

This transfer function is used to tune the PI control. The discretized PI controller function, given by the method of backward difference, is given by the expression in Eq. 5, and its discrete equivalent is given in Eq. 6.

$$
\begin{gathered}
\frac{u^{\prime}(z)}{e(z)}=P I(z)=K p\left(1+\frac{T_{s}}{T i} \frac{1}{1-z^{-1}}\right) \\
u^{\prime}(k)=P I(z)=K p \cdot\left(e(k)-e(k-1)+K p \cdot \frac{T_{S}}{T i} e(k)+u^{\prime}(k-1)\right.
\end{gathered}
$$

The resulting equations for the PI control are expressed in Eq. 7.

$$
\begin{aligned}
& u_{d}(k)=K p \cdot\left(e_{d}(k)-e_{d}(k-1)+K p \cdot \frac{T_{S}}{T i} e_{d}(k)-\omega L \cdot i_{q}(k)+v_{d}(k)+\omega L \cdot i_{q}(k-1)-v_{d}(k-1)\right. \\
& u_{q}(k)=K p \cdot\left(e_{q}(k)-e_{q}(k-1)+K p \cdot \frac{T_{S}}{T i} e_{q}(k)+\omega L \cdot i_{d}(k)+v_{q}(k)-\omega L \cdot i_{d}(k-1)-v_{q}(k-1)\right.
\end{aligned}
$$

\section{Simulation}

Figure 5 depicts a simulation example of this implementation, displaying the reference tracking of $i_{d}$ and $\mathrm{i}_{\mathrm{q}}$ currents, obtained from the active and reactive power references.
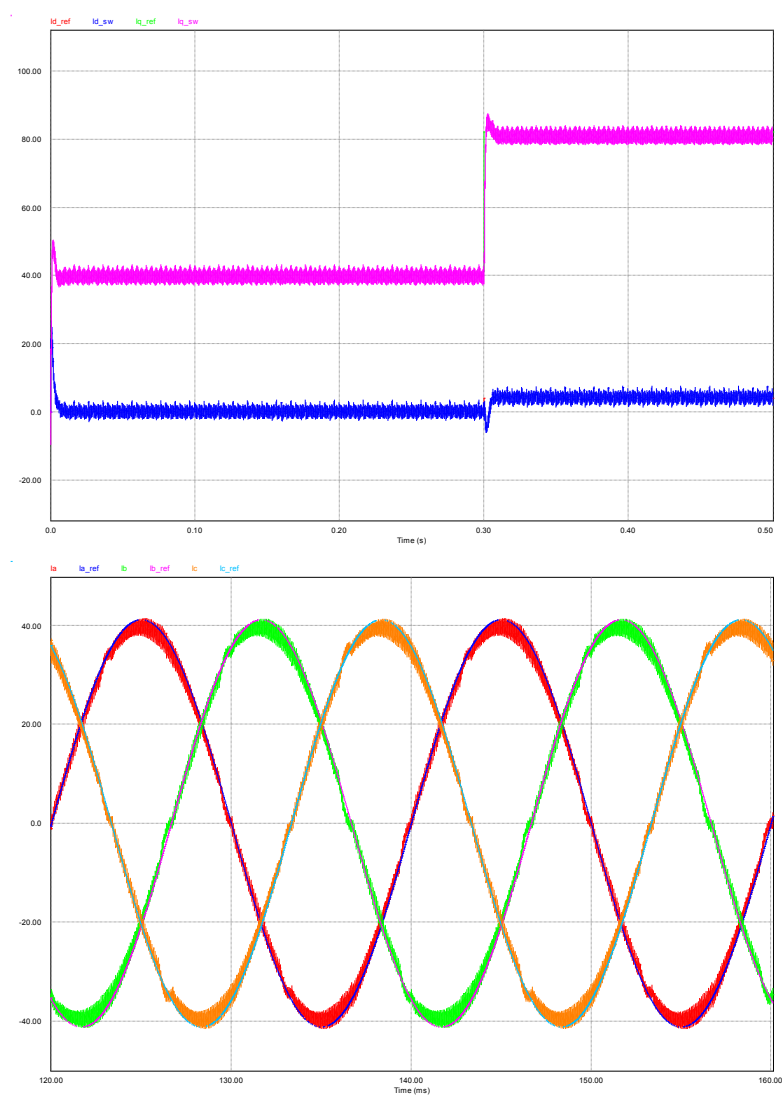

Fig. 5. Simulation example for decoupled PI control

Total harmonic distortion is THD $=3.5217 \%$. The initial overshoot is reduced by means of decoupling and disturbance compensation but THD becomes worse with respect to the initial PI with no compensation.

\subsection{Dead Beat with integral action}

The Dead Beat control belongs to the category of predictive digital controllers, since the output voltage is computed to make the current to follow a predictive model-based reference. It can be seen as the inverse digital control of the closed-loop model. Presents a fast dynamic response, assuring that any error vector different from zero will be conducted to zero in a given number of samples. The control block diagram of this controller can be seen in Fig. 6.

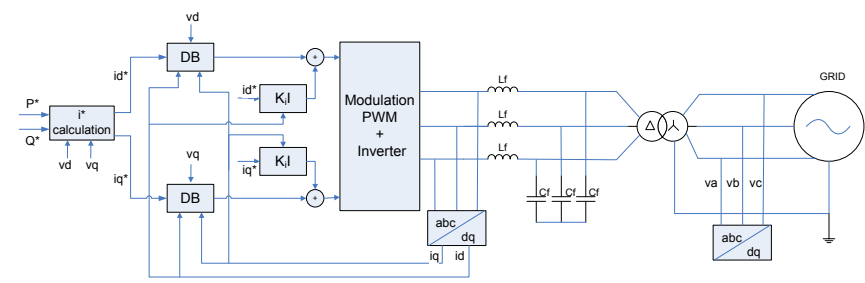

Fig. 6. Block diagram of dead beat controller with integral action.

The dead beat control action can be straightforward computed from the state space discrete description of Eq. 2. The control laws are derived in Eq. 8. 


$$
\begin{aligned}
& u_{d}(k)=\frac{1}{b_{11}} i_{d R E F}(k+1)-\frac{a_{11}}{b_{11}} i_{d}(k) \underbrace{-\frac{a_{12}}{b_{11}} i_{q}(k)+v_{d}(k)}_{u \operatorname{dec}(k)} \\
& u_{q}(k)=\frac{1}{b_{22}} i_{q R E F}(k+1)-\frac{a_{22}}{b_{22}} i_{d}(k) \underbrace{-\frac{a_{21}}{b_{22}} i_{q}(k)+v_{d}(k)}_{\text {udec }(k)}
\end{aligned}
$$

The parameter values are the following: a11 $1-\mathrm{T}_{\mathrm{S}} / \mathrm{L}$, $\mathrm{a} 12=\mathrm{T}_{\mathrm{S}} \mathrm{w}, \mathrm{a} 21=-\mathrm{T}_{\mathrm{S}} \mathrm{w}, \mathrm{a} 22=1-\mathrm{T}_{\mathrm{S}} / \mathrm{L}$ and $\mathrm{b}=\mathrm{T}_{\mathrm{S}} / \mathrm{L}$. The control decoupling terms $\mathrm{u}_{\mathrm{dec}}$ are explicitly included in the discrete control law. Considering that no modeling errors are found, the closed loop response is equivalent to the reference value (Eq. 9).

$$
i(k+1)=i_{R E F}(k)
$$

It is well known that the dead beat control provides a fast dynamic response, but the setpoint tracking and stability are not guaranteed in presence of model uncertainties. Therefore, since a null model uncertainty should not be assumed, the dead beat control alone is not used, but additional plug-in controllers are included in addition, to compensate unmodelled dynamics. In this case an integral action [3] is added to compensate for uncertainty and to achieve steady state error cancellation.

\section{Integral action}

If it is assumed that the closed loop response follows the reference with a unit sample delay, then adding an integral action considers the error between the reference signal and one sample delayed output. Thus the discrete control law for integral action follows as written in Eqs. 10 and 11.

$$
P(s)=\frac{1}{s L+R}
$$

$$
u I(k+1)=u I(k)+K i T_{S}\left(i_{R E F}(k-1)-i(k)\right)
$$

The total control laws for each axis ( $d$ and $q$ ) are given by Eq. 12.

$$
\begin{aligned}
& u_{d}(k)=\frac{1}{b_{11}} i_{d R E F}(k+1)-\frac{a_{11}}{b_{11}} i_{d}(k) \underbrace{-\frac{a_{12}}{b_{11}} i_{q}(k)+v_{d}(k)}_{u \text { dec }(k)}+u I_{d}(k) \\
& u_{q}(k)=\frac{1}{b_{22}} i_{q R E F}(k+1)-\frac{a_{22}}{b_{22}} i_{d}(k) \underbrace{-\frac{a_{21}}{b_{22}} i_{q}(k)+v_{d}(k)+u I_{q}(k)}_{\text {udec }(k)}
\end{aligned}
$$

\section{Simulation}

Figure 7 depicts a simulation example of this implementation, displaying the reference tracking of $i_{d}$ and $\mathrm{i}_{\mathrm{q}}$ currents, obtained from the active and reactive power references.
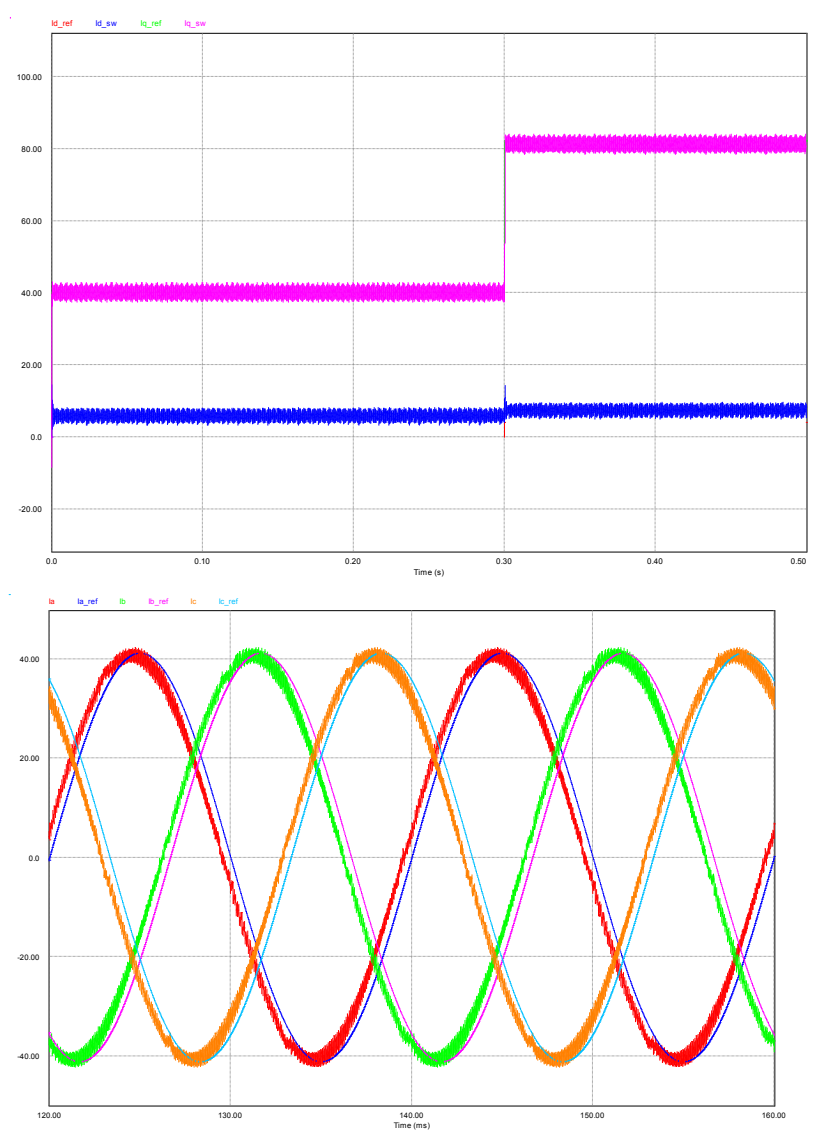

Fig. 7. Simulation example of the deadbeat control
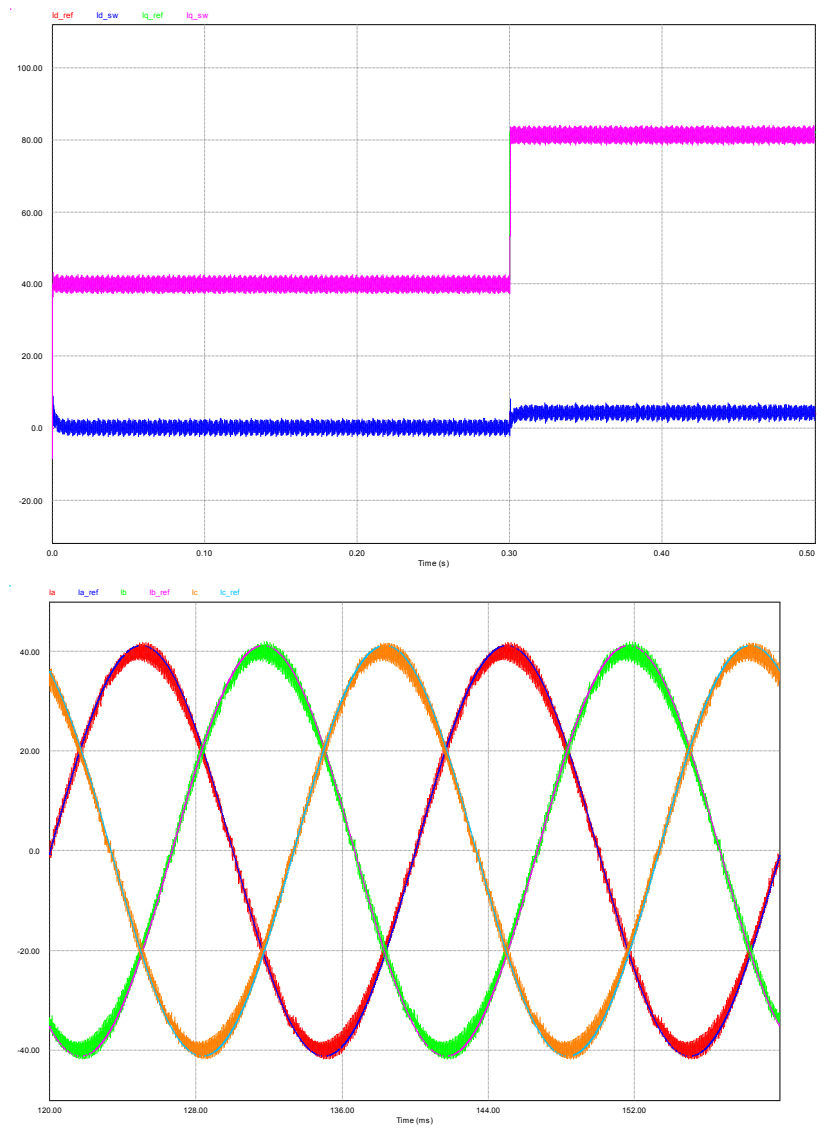

Fig. 8. Simulation example of the deadbeat control with integral action

Dead Beat control presents a position error on $\mathrm{dq}$ coordinates and therefore a phase error in natural 
coordinates. Adding an integral action and properly tuning the gain removes the tracking error. Total harmonic distortion is THD $=3.3772 \% \%$.

\subsection{Proportional Resonant Control}

The resonant control is based on the use of the generalized integrator (GI). It is basically using a double integrator that achieves infinite gain in the open loop at the selected frequency (resonance frequency) and attenuation just outside. Fig. 8 depicts the block diagram of this control.

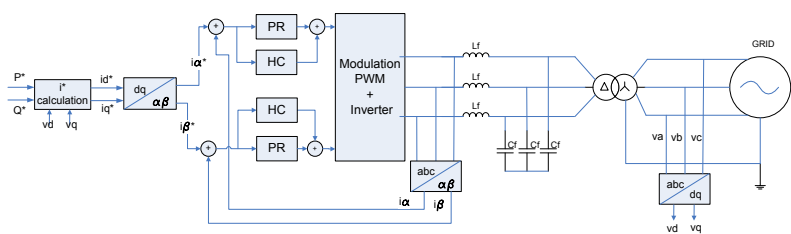

Fig. 9. Block diagram of the proportional resonant controller.

Due to the structure of this control, the dq-Park transformations are no longer necessary, and the control design can be done in natural reference frame $(a b c)$ or in stationary reference frame control $(\alpha \beta)$. Since there are no coupled terms in these reference frames, no decoupling control terms are necessary either. Moreover, due to the intrinsic capability of this control to achieve high gains at the selected frequency, zero steady state error and grid voltage disturbance rejection are guaranteed.

The abc- $\alpha \beta$ transformation is chosen for the design, so that the three phase system turn in two independent single-phase systems axes which allow tuning just two controllers instead of three. The controller transfer functions matrix is written in Eq. 13.

$$
G_{P R}{ }^{(\alpha \beta)}(s)=\left[\begin{array}{cc}
K_{p}+\frac{K_{i} s}{s^{2}+w_{0}{ }^{2}} & 0 \\
0 & K_{p}+\frac{K_{i} s}{s^{2}+w_{0}{ }^{2}}
\end{array}\right]
$$

Compensation of harmonics is achieved by simply cascading more generalized integrators tuned to resonate at selected harmonic frequencies, with the transfer function written in Eq. 14.

$$
G_{h}(s)=\frac{K_{i} s}{s^{2}+w_{h}^{2}}
$$

Tuning this controller is the only task to carry out. The Matlab's SISOTOOL application has been used. If the converter-side current is sensed, the plant transfer function is as can be seen in Eq. 15, where $\mathrm{z}_{\mathrm{LC}}^{2}=\left[\mathrm{L}_{\mathrm{G}} \mathrm{C}_{\mathrm{f}}\right]^{-1}$ and $\mathrm{w}_{\text {res }}^{2}=\left(\mathrm{L}_{\mathrm{f}}+\mathrm{L}_{\mathrm{G}}\right) \cdot \mathrm{z}_{\mathrm{LC}}^{2} / \mathrm{L}_{\mathrm{f}}$.

$$
G_{f}(s)=\frac{i(s)}{u(s)}=\frac{s^{2}+z^{2} L C}{L_{f}\left(s^{2}+\omega^{2}{ }_{r e s}\right)}
$$

\section{Simulation}

Figure 9 depicts a simulation example of this implementation, displaying the reference tracking in the $\alpha \beta$ currents, obtained from the active and reactive power reference values.
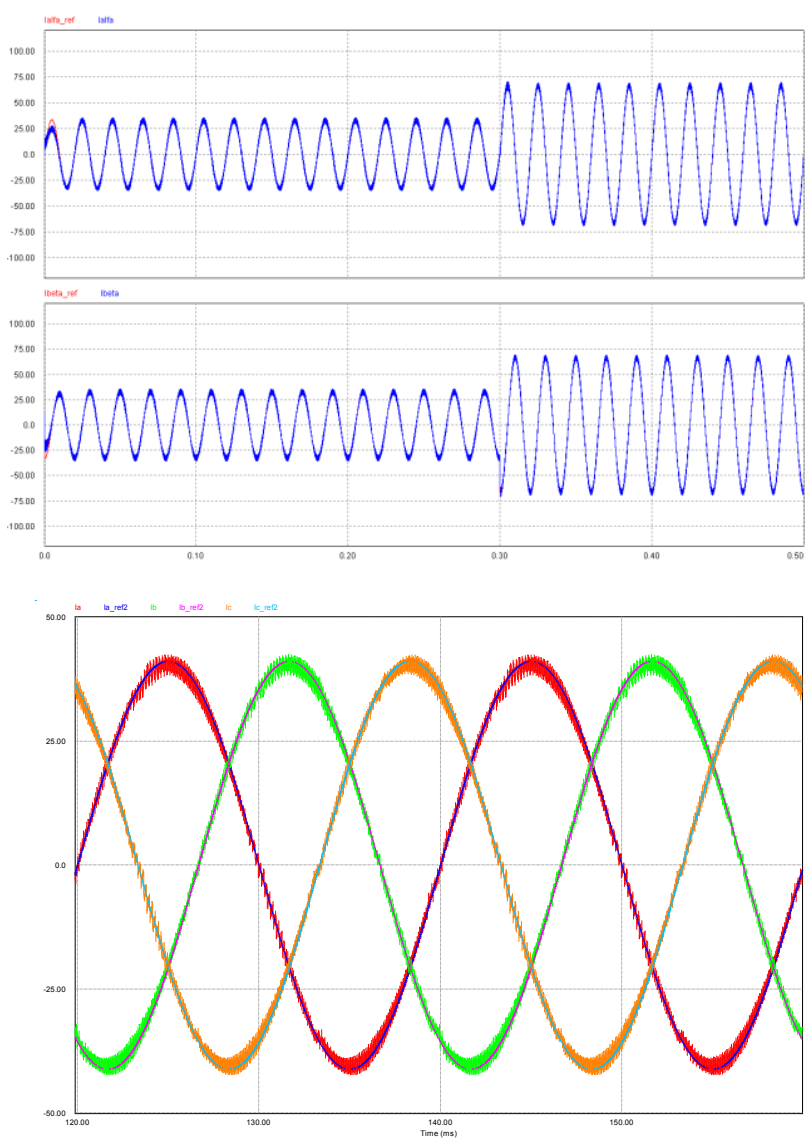

Fig. 10. Simulation example of the PR controller.

In natural coordinates under steady state conditions some distortion can be appreciated in the peaks of the wave.

Total harmonic distortion is $\mathrm{THD}=3.4432 \%$.

\section{Conclusions and Future Works}

The controllers designed show in general similar performance. Assuming no computational delay, the frequency response is similar, finding no significant difference in the elimination of low frequency harmonics and in THD, in any case attributable to a better or worse tuning of the parameters of each controller.

In the PI control there is always a phase error which is more apparent in the zero crossing and on slope changes of the sine wave. Besides, changes in current set points show high initial overshoots even in opposite direction due to the cross-coupling. This initial overshoot is reduced adding feedforward compensation of crosscoupling and disturbance but no significant improvement on THD is detected. 
The lowest harmonic distortion for the selected time window is achieved by Dead Beat control. Also a better damping with little overshoot at a simulation stage is observed. However, due to unmodelled dynamics there is a tracking error which can be corrected by adding integral action. Furthermore, in presence of computational delay, the dead beat controller shows the highest worsening in the response.

Proportional Resonant control presents the better balance in terms of THD, robustness and tracking of current references. Furthermore it has the easiest implementation, proper tuning of the proportional and integral gains makes unnecessary to include feedforward compensation terms or decoupling as in the case of the IP, and adding terms to maintain performance in the presence of uncertainty, as in the case of Dead Beat control.

In presence of computational delay, an increase of THD is appreciated in all cases although is not very significant, but oscillations start to appear. This can be compensated if the computational delay is considered in the controllers' design, predicting the control action to be applied in the next period, but this requires the design and tuning of observers/predictors, complicating the control algorithms.

It must be highlighted that integral action can produce instability or a very large deterioration in the current wave, increasing the ripple, and therefore the THD, which would result impractical from the point of view of wave quality injected into grid. This is due to the known increase in the phase shift and the effect of wind-up caused by saturation in the control actions of integral control [7]. This effect becomes more apparent in presence of computational delays.

Further issues have still to be considered, and the systems have to be programmed in the DSP and tested at the inverter. Future works in this development will also include taking into account computational delays in the design, and uncertainty robustness. Furthermore, other control techniques combinations are being evaluated, as cascaded PI controllers with dead beat response [8] and dead beat control with plug-in repetitive controller [4].

\section{Acknowledgement}

The development of this work has been supported by the IMPIVA (Institute of the Small and Medium-sized Industry), which is attached to the Conselleria de Industria, Comercio e Innovación (Industry, Trade and Innovation Regional Minister) of the Valencia Regional Government, and the European Regional Development Fund (ERDF) from the European Comission.

\section{References}

[1] Frede Blaabjerg, Remus Teodorescu, Marco Liserre and Adrian V. Timbus. Overview of Control and Grid Synchronization for Distributed Power Generation Systems. IEEE Transactions On Industrial Electronics, Vol. 53, No. 5, October 2006

[2] Pedro Roncero-Sanchez, Vicente Feliu and Aurelio Garcia-Cerrada. Predictive-Integral Current Controller for Active- and Reactive-Power Control of Wind Generators.

[3] A new control structure for grid-connected LCL PV inverters with zero steady-state error and selective harmonic compensation. Teodorescu, R., Blaabjerg, F., Borup, U., Liserre, M. Applied Power Electronics Conference and Exposition, 2004. APEC Volume 1, Issue , 2004 Page(s): 580 - 586 Vol.1

[4] Digital Repetitive Controlled Three-Phase PWM Rectifier. Keliang Zhou and Danwei Wan,. IEEE Transactions On Power Electronics, Vol. 18, No. 1, January 2003

[5] Milan Prodanovic and Timothy C. Green, Member. Control and Filter Design of Three-Phase Inverters for High Power Quality Grid Connection. IEEE Transactions On Power Electronics, Vol. 18, No. 1, January 2003

[6] Entendiendo la Transformación de Park. Francisco M. Gonzalez-Longatt, Junio 2004. In Spanish.

[7] Control System Design. Graham C. Goodwin, Stefan F. Graebe, Mario E. Salgado

[8] Current Control of Voltage Source Converters connected to the grid through an LCL-filter. Emilio. J. Bueno, Felipe Espinosa, Francisco J. Rodríguez, Jesús Ureña, Santiago Cobreces 